\title{
ON THE VALIDITY OF THE SLIT ISLANDS ANALYSIS IN THE MEASURE OF FRACTAL DIMENSION OF FRACTURE SURFACES
}

\section{Chunsheng $\mathrm{Lu}$}

Laboratory for Nonlinear Mechanics of Continuous Media

Institute of Mechanics, Academia Sinica

Beijing 100080, Peoples's Republic of China

Fax: (86-1) 2561284

Slit islands analysis (SIA) for the measure of the fractal dimension of fracture surfaces introduced by Mandelbrot et al. [1] has been widely used in fractography of fracture surfaces. At the same time, the validity of fractal dimension measured with SIA method has still been in dispute [2-6]. This method is based on the following relation

$$
P(\delta)^{1 / D}=\alpha(\delta) \quad A(\delta)^{1 / 2}
$$

where $\mathrm{P}$ and $\mathrm{A}$ are the perimeter and the area of islands which is formed when a specimen plated with nickel is polished parallel to the plane of fracture individually, $\delta$ is the yardstick used for measuring, $D+1$ is the fractal dimension of the fracture surface.

In the case of standard shapes $(D=1)$, the ratio $\alpha(\delta)$ between perimeter and area is a dimensionless constant. As an example, for a circle, $\alpha=2 \sqrt{\pi}$. However, for nonstandard or fractal shapes, the ratio $\alpha(\delta)$ is obviously related to the yardstick $\delta$, that is

$$
\alpha(\delta)=\frac{[P(\delta)]^{1 / D}}{[A(\delta)]^{1 / 2}}
$$

According to fractal theory [7], the length of a fractal curve is

$$
P(\delta)=N(\delta) \quad \delta=\left(\frac{\delta}{P_{0}}\right)^{-D} \delta=P_{0}^{D} \quad \delta^{1-D}
$$

where $N(\delta)$ is the number measured with the yardstck $\delta, P_{0}$ is the characteristic length of the fractal curve. Here $P_{0}$ is the length of the initiator. It is worth noting 
that $P(\delta)$ in (3) is the measured perimeter and its dimension is still [length]. In the application of fractal geometry, the physical dimension should remain unchangeable.

Combining (2) and (3), we obtain

$$
\alpha(\delta)=\frac{P_{0} \delta^{(1-D) D}}{[A(\delta)]^{1 / 2}}
$$

When we use the SIA method to measure the fractal dimension of fracture surfaces, the yardstick $\delta$ is a fixed value. Intuitively, the ratio $\alpha(\delta)$ seems to relate to the size of islands. In fact, Mandelbrot has shown that the ratio $\alpha(\delta)$ is independent of the shapes and sizes of fractals [7].

Suppose that we have a series of Koch islands with different sizes in a section surface. For the $i$ th Koch island (see Fig. 1), its size can be determined by its initial lateral length a. Then its perimeter and area are individually

$$
\begin{aligned}
& P(\delta)=3(4 \delta)^{n} a \\
& A(\delta)=\frac{\sqrt{3}}{4}\left\{1+\frac{3}{5}\left(1-\left(4 \delta^{2}\right)^{n}\right)\right\} a^{2}
\end{aligned}
$$

where $\mathrm{n}$ is the number of self-similar levels related to the yardstck $\delta$, here, $\delta=(1 / 3)^{\mathrm{m}}$. The initial perimeter $P_{0}$ of the $i$ th Koch island is equal to 3a. From (4), we have

$$
\alpha(\delta)=\frac{P_{0} \delta^{(1-D) / D}}{[A(\delta)]^{1 / 2}}=\frac{3 \delta^{(1-D) / D}}{\sqrt{\frac{\sqrt{3}}{4}\left\{1+\frac{3}{5}\left(1-\left(4 \delta^{2}\right)^{n}\right)\right\}}}
$$

It is obvious that the ratio $\alpha(\delta)$ is only dependent on the yardstick $\delta$, and independent of the size of the islands. At the same time, we also see that the ratio $\alpha(\delta)$ is dependent on the self-similar level, that is, the scaling range when the yardstick $\delta$ is fixed.

Generally speaking, the value of area $A(\delta)$ increases much slower than that of its perimeter $\mathrm{P}(\delta)$ as the yardstick $\delta$ decreases. In most cases, the $\mathrm{A}(\delta)$ almost tends to a constant if small $\delta$ is chosen or there is a certain range of scaling. Then

$$
A(\delta) \sim<d>^{2}
$$

where $<\mathrm{d}\rangle$ is the average diameter of the island. Therefore, the relation (1) can be rewritten as 


$$
P(\delta)^{1 / D} \sim<d>
$$

Recently, Mu et al. [8] pointed out a similar method, perimeter and maximum-diameter relation, which can well estimate the fractal dimension of fracture surfaces. In order to ensure the validity of (9), a certain scaling range is needed. In our opinion, the scaling range must be over two orders of magnitude.

According to the discussion above, we can see that the statistical self-similarity of the islands in fracture section is a prerequisite condition. However, it is often very difficult to evidence the self-similarity a priori. Considering the anisotropy of microstructures in materials and outer loading, it is possible that the fracture surfaces are self-affine [9], rather than self-similar. There is now sample evidence that fracture surfaces possess statistically self-affine fractal properties $[10,11]$. Therefore, the neglect of this geometrical feature can also result in various disputes about the true value of the fractal dimension $\mathrm{D}$, and even obtain the claims that D can take any of two or more values for the same fractal [12].

In conclusion, the fractal dimension of fracture surfaces can be well estimated by the SIA method if the fracture surface is statistically self-similar and a certain range of length exists. At the same time, an equivalent and easily measured method, perimeter and average-diameter relation, is given.

Acknowledgement: This work is supported by the National Natural Science Foundation of China under Grant No. 19302021 and by the National Basic Research Project "Nonlinear Science".

\section{REFERENCES}

[1] B.B. Mandelbrot, D.E. Passoja and A.J. Paullay, Nature 308 (1984) 721-722.

[2] E. Underwood and K. Banerji, Materials Science and Engineering 80 (1986) $1-14$.

[3] C.S. Pande, L.E. Richards, N. Louat, B.D. Dempsey and A.J.Schwoeble, Acta Metallurgica 35 (1987) 1633-1637.

[4] C.W. Lung and Z.Q. Mu, Physics Review B, 38 (1988) 11781-11784.

[5] L.V. Meisel, Journal of Physics D: Applied Physics 24 (1991) 942-952.

[6] W. Lei and B. Chen, International Journal of Fracture 65 (1994) R29-R39.

[7] B.B. Mandelbrot, The Fractal Geometry of Nature, Freeman, San Francisco (1982).

[8] Z.Q. Mu, C.W. Lung, Y. Kang and Q.Y. Long, Physical Review B, 48 (1993) 7679-7681. 
[9] B.B. Mandelbrot, Physica Scripta 32 (1985) 257-260.

[10] K.J. Maloy, A. Hansen and E.L. Hinrichsen, Physical Review Letters 68 (1992) 213-215.

[11] J.P. Bouchaud, E. Bouchaud, G. Lapasset and J. Planes, Physical Review Letters 71 (1993) 2240-2243.

[12] B.B. Mandelbrot, Fractals 1 (1993) 117-123.

29 November 1994

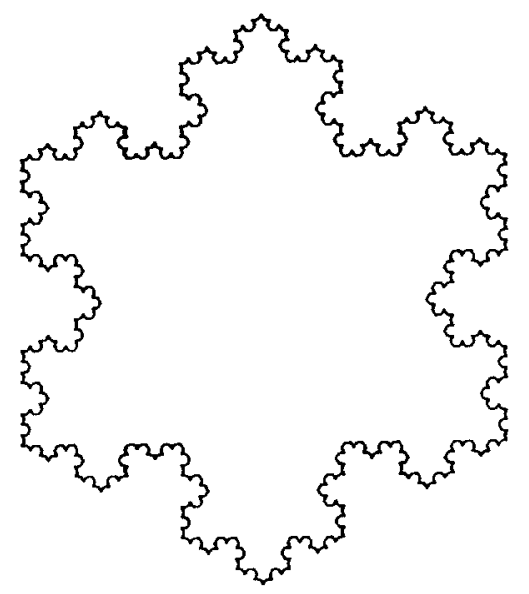

Figure 1. The triadic Koch island. 